\title{
Optimizing use of opiates in the management of cancer pain
}

\author{
Mario Mandalà' \\ Cecilia Moro' \\ Roberto Labianca' \\ Marco Cremonesi ${ }^{2}$ \\ Sandro Barni \\ 'Unit of Medical Oncology, Ospedali \\ Riuniti di Bergamo; ${ }^{2}$ Division of \\ Medical Oncology, Treviglio Hospital, \\ Bergamo, Italy
}

\begin{abstract}
Cancer pain is often suboptimally managed. The underestimation and undertreatment continues to be a problem despite the availability of consensus-based guidelines. Most patients with cancer develop pain. The prevalence and severity of pain among cancer patients varies according to primary and metastatic sites and stage of disease. Opioid therapy is the cornerstone of management of severe chronic pain in the field of cancer patients and in general in palliative care medicine. Since this class of drugs is the cornerstone of the treatment, optimizing its use may be useful in clinical practice. For this purpose we focused on 4 distinct issues: 1) How to implement the use the opioids in cancer patients; 2) How to optimise the use of morphine in cancer patients; 3 ) The management of side effects and opioid switching; 4) What is the role of other potent opioids. A holistic approach including an appropriate use of opioids may improve pain control in most cancer patients, particularly for those with advanced disease.
\end{abstract}

Keywords: opioids, pain, cancer patients

\section{Introduction}

Most patients with cancer develop pain. The prevalence and severity of pain among cancer patients varies according to primary and metastatic sites and stage of disease. At least 30\% of cancer patients receiving active treatment for metastatic disease have significant cancer-related pain, while this percentage increases to between $60 \%$ and $90 \%$ in those with advanced disease (Levy and Samuel 2005).

Opioid therapy is the cornerstone of management of severe chronic pain in the field of cancer patients and in general in palliative care medicine (Hanks et al 2001; Davis et al 2005).

Cancer pain is often suboptimally managed. The underestimation and undertreatment continues to be a problem despite the availability of consensus-based guidelines.

Healthcare professionals may be overly concerned about the development of analgesic tolerance, side-effect management, and regulatory concerns. They may not be aware of current knowledge concerning the mechanisms of pain and the modalities available for its control. Similarly, patients may be concerned about side effects, tolerance and the untrue thought that in patients with cancer pain is an inevitable symptom (Davis et al 2005).

Current pain treatment is based on the World Health Organization (WHO) threestep analgesic ladder. The WHO ladder has been extensively validated (Hanks et al 2001). It involves a stepwise approach to the use of analgesic drugs. The philosophy behind the WHO ladder is to give framework principles, which allows flexibility in the choice of analgesics. Potent opioids are the analgesics of choice as the third step of the WHO guideline. Opioids should be given "by the mouth, by the clock, by the ladder". Since this class of drugs is the cornerstone of the treatment, optimizing its use may be useful in clinical practice. 
For this purpose we focused on 4 distinct issues: 1) How to implement the use the opioids in cancer patients; 2) How to optimize the use of morphine in cancer patients; 3 ) The management of side effects and opioid switching; 4) What is the role of other potent opioids?

\section{How to implement the use the opioids in cancer patients}

There are at two issues, which should be considered:

1) All patients should receive an appropriate assessment, including screening for the presence of pain, and when it is present it is necessary a comprehensive evaluation. Pain assessment should include the "intensity" of pain, "how" and "when" it manifests and disappears, the location and quality of pain, and aggravating and alleviating factors. It is crucial to evaluate previous treatments and their effectiveness. It is important to establish the impact of pain on physical and emotional function. This approach needs an interdisciplinary, collaborative care planning. The interdisciplinary team should bring together individuals with a diversity of training, who share the goal of "an holistic pain control". The members of the team should include the physicians, nurses, social workers, physiotherapist, occupational therapists, dietician, pharmacist, volunteer, and in particular situations, the chaplain, the music and the art therapist (Gordon et al 2005). Most importantly, patients and family should be involved to improve compliance and provide information about available pain control options. It is important to tailor treatments and identify the needs and desires of our patients. Trowbridge and colleagues (1997) found that standardized pain assessment alone improved cancer pain management and reduced patient-reported pain severity.

2) Further improvement in symptom management might be achieved with simple protocols or guidelines for symptom management. For example, Du Pen and colleagues (1999) found that the institutional use of a simple protocol for cancer pain management reduced pain severity. Similarly Cleeland and colleagues (2005) suggested that a protocol for cancer pain management can improve pain control.

\section{How to optimize the use of morphine in cancer patients}

Morphine is a cornerstone for the management of pain in cancer patients (Hanks et al 2001). There is no proven superiority of morphine as compared with other available opioids. However morphine is the most used opioid and this is due to extensive experience among physicians, a large variety of formulations and no clinically relevant ceiling effect to analgesia. Morphine is also the most cost-effective analgesic. However morphine has some limitation. The systemic availability of morphine by the oral route is poor and this may cause a great inter-individual variability in doses appropriate for an adequate response. In addition active metabolites may contribute to toxicity, especially for patients with renal impairment.

The oral route is the simplest and of choice for administering morphine. The dose of morphine should be titrated with a normal release formulation given every 4 hours. The titration with normal morphine helps to rapidly achieve the steady state. In addition this strategy helps to adjust promptly the dose during the dose finding period. If the pain is not controlled during the dose titration, a gradually increase of the dose by $50 \%$ may be recommended: for example patients who begin with $10 \mathrm{mg}$ may receive first $15 \mathrm{mg}$ and then $20 \mathrm{mg}$ until an active dose is reached. The starting dose depends by previous treatments: patients coming from step two will start with $10 \mathrm{mg}$ of normal morphine preparation every 4 hours; on the contrary if the step two is omitted, $5 \mathrm{mg}$ of normal morphine preparation every 4 hours may be an adequate dose for the beginning of titration. During the titration period morphine should be given every 4 hours and a full 4-hourly dose should be used for rescue. Consequently the rescue dose should be increase at the same time and to the same level in line with the regular four hourly dose. In the general practice once the patient's pain has been controlled and a stable dose is achieved for 48 hours, the regular dose may be converted to an oral controlled-release (CR) preparation. In several controlled clinical trials, investigating the management of pain in cancer patients, CR morphine given every 12 hours provided effective analgesia with a discontinuation rate of only $2 \%$. From a practical point of view, it is important to provide an immediate release (IR) morphine preparation to patients stabilized on CR morphine to be used "as required" for incidental pain. For patients who are unable to take oral morphine, the preferred alternative route to administer morphine is the subcutaneous way. The peak concentrations are achieved within 15-30 minutes, and the onset of action is more rapid than that achieved with the oral formulation. Subcutaneous morphine does not undergo first pass metabolism. To convert a 24 hour dose of oral morphine to an equivalent subcutaneous, the dose of oral morphine 
should be divided by three to achieve an equianalgesic effect due to the patency ratio of nearly $1: 3$. For example, $90 \mathrm{mg}$ of oral sustained morphine release twice daily is equivalent to $60 \mathrm{mg}$ subcutaneous morphine. In patients with breakthrough pain, subcutaneous morphine injection equivalent to a 4 hourly morphine dose should be considered.

The role of intravenous morphine is more limited than the oral or subcutaneous infusion. Intravenous morphine may be considered for patients with a central venous access, generalized oedema, or with complications due to local intradermal infusion (Hanks et al 2001).

Finally there is controversy about the role of spinal morphine administration. Smith and colleagues (2005) suggested that spinal opioids may sustain pain control, less drug-related toxicity, and possibly better survival (Smith et al 2002, 2005). However the above mentioned studies have important limitations (Davis et al 2003; Ripamonti and Brunelli 2003) In addition three studies investigated the early use of such type of administration, all of which found no advantage (Vainio and Tigerstedt 1988; Sjogren and Banning 1989; Kalso et al 1996). The European Association for Palliative Care (EAPC) stated that few patients need spinal opioids (Hanks et al 2001). This type of administration should be considered in the niche of patients who received pain control with intolerable side effects, or when pain control has been unsatisfactory with opioid rotation. If patients receive adequate opioids early, only a small subset will need spinal opioids.

\section{The management of side effects}

Morphine is generally well tolerated, it has been estimated that $10 \%-30 \%$ of patients treated with oral morphine do not have a successful outcome because of excessive adverse effects or inadequate analgesia (Cherny et al 2001).

The management of adverse effects remains a major clinical challenge, especially because the lack of studies comparing various therapeutic approaches able to counteract the side effects. The first step in the evaluation of patients is to differentiate the adverse effects from other causes such as hystopathology of the tumor, sites of the disease, concomitant medication and comorbidity, which may mimic opioid-induced adverse effects. Patients with cerebral or leptomeningeal metastases, dehydration, hypercalcemia, hyponatriemia, renal or liver failure may have drowsiness and cognitive impairment. Similarly concomitant chemotherapy or radiotherapy, or both, which can cause bowel obstruction, may mimic opioid-induced nausea or vomiting.

If external factors, which may mimic opioid-induced adverse events, are reasonably excluded, physicians should consider several approaches to the management of opioid adverse effects: 1) a reduction of opioid dose; 2) the symptomatic management of the opioid toxicity; 3 ) opioid rotation, also defined as opioid switching; 4) switching the route of administration.

It may be difficult to reduce the dose of opioids for patients with a well controlled pain, because of the loss of pain control. In these cases, several strategies may be applied: administration of non-opioid coanalgesic; the addition of adjuvant analgesic; administration of appropriate palliative chemotherapy, radiotherapy or surgery. These approaches have several limitations and should be tailored to the single patient. It is known that non-steroidal antiinflammatory agents may cause additive toxicities in patients treated with opioids. Cancer patients receiving chemotherapy such as cisplatin may be at risk of developing renal toxicity. This risk may be increased by the concomitant use of nonsteroidal anti-inflammatory drugs (NSAIDs). They may provide additive pain relief for some patients who already are receiving opioids for moderate to severe cancer pain. According to a recent Cochrane review, most studies reporting safety data of opioids in combination with NSAIDs are small and heterogeneous in terms of type of cancer or cancer pain, level of pain among participants, and specific medications used. In addition, none of the studies lasted more than two weeks, so potential adverse effects from prolonged use of these treatments individually or in combination could not be assessed or compared (McNicol et al 2005.).

Furthermore, patients with neuropathic pain drugs such as gabapentin may cause drowsiness and cognitive impairment in the titration phase, with may be additive to opioids. Chemotherapy and radiotherapy may increase nausea, vomiting, or cognitive impairment. Finally pharmacokinetic interaction between opioids and chemotherapic agents may increase the toxicity of opioids.

\section{Gastrointestinal side effects}

Nausea and vomiting are common during the titration phase, tolerance over time, so subsequently may disappear without therapy (Walsh et al 1992; Cherny et al 2001). Several agents have been suggested for patients with persistent nausea and vomiting: metoclopramide, 5-HT3 antagonists, haloperidol, 
corticosteroids, or phenothiazine. There is not evidence suggesting the superiority of one antiemetic over another.

On variance, constipation shows no tolerance over time. This type of side effect has been reported in $40 \%$ to $70 \%$ of patients receiving morphine for chronic pain. Several agents have been suggested to counteract this side effect: senna, bisacodyl, phenolphthalein, and lactulose. As for nausea and vomiting there is no evidence to suggest the superiority of one laxative over another.

\section{Central nervous system side effects}

As for some gastrointestinal side effects like nausea and vomiting, a transient cognitive impairment is common after the initiation of opioid therapy. Sedation, the most commonly seen symptom of toxicity, is considered the "tip of the iceberg" leading toward delirium and obtundation (Bruera et al 1989, 1992). When sedation occurs, other causes need to be excluded, and one can wait 7 to 10 days to allow tolerance to develop. One should also discontinue other sedating adjuvant medications. If symptoms persist, the next step is opioid dose reduction or opioid rotation to an alternate drug or another route of administration. When evaluating the patient with delirium, it is important to consider nonopioid causes, including infection, electrolyte imbalance, and other potentially neurotoxic medications commonly used as adjuvants, such as the antidepressants and anticonvulsants. It is prudent to discontinue all benzodiazepines, and consider a trial of hydration. Opioidinduced myoclonus is often a sign of evolving delirium. Risk factors include dehydration and other medications, such as selective serotonin reuptake inhibitors, dopamine antagonists, and NSAIDs. These drugs (NSAIDs) are thought to produce mild renal insufficiency and decrease opioid and metabolite excretion. Treatments to consider are hydration, opioid rotation, and GABA agonists such as clonazepam and baclofen.

For severe opioids-induced cognitive impairment, for example delirium, neuroleptics, specifically haloperidol, are the most recommended drugs. For patients with delirium and severe agitation, a treatment with neuroleptics such as co-administered haloperidol and benzodiazepine is suggested (Battaglia et al 1997). An improvement of agitated confusion even after opioid rotation was reported in the literature (Paix et al 1995; Ashby et al 1999).

Patients with severe agitation are often co-administered a benzodiazepine (Battaglia et al 1997). This latter class of drugs have been recommended for myoclonus. Several reports have reported a role for diazepam, clonazepam, midazolam, and baclofen (De Conno et al 1991; Eisele et al 1992; Obeso 1995). Unfortunately there are no prospective trials on the treatment of opioid-induced myoclonus.

\section{Opioid switching to improve pain relief and drug tolerability}

There are several clinical experiences suggesting that a failure to respond to one opioid does not mean failure to respond to all opioids (Mercadante 1999). Although there are different biological explanations behind this consideration, the true reason why opioid substitution is successful remains unclear. Pharmakogenetic, pharmakogenomic, pain mechanism, and degree of cross tolerance should be considered, but it is reasonable to think that perhaps there is no single specific explanation. Every user of opioids is aware that poorly responsive pain may arise because of the development of analgesic tolerance, while no tolerance to adverse effects develops to the same extent. This means that the escalating dose of any opioid may reach a level at which there is a detrimental balance between analgesia and side effects.

The term opioid rotation has been reported in literature. This implies that there is a rotation back to the original opioid after switching. As very few reports reported a true opioid rotation, the term opioid switching may be more appropriate in clinical setting, because it reflects more strictly the clinical study results. Different strategies have been reported, including switching from oral or subcutaneous morphine to methadone, fentanyl to methadone, and morphine to oxicontin. As regards the switching from morphine to methadone, a previous report by Ripamonti and colleagues (1998) showed a dose ratio of 1:4 (1 mg of oral methadone $=4 \mathrm{mg}$ of oral morphine) for patients receiving less than $90 \mathrm{mg}$ of morphine, while a ratio of 1:8 and 1:12 has been suggested for those patients receiving $90 \mathrm{mg} / \mathrm{day}$ to $300 \mathrm{mg} /$ day and greater than $300 \mathrm{mg} /$ day respectively (Ripamonti et al 1998). However in patients with uncontrolled pain, a starting dose of methadone increased by approximately $20 \%-30 \%$ has been suggested (Mercadante et al 2001). It is reasonable to think that future studies are needed in order to better define the dose ratio between morphine and methadone.

Recently Riley and colleagues (2006) reported a prospective, observational, controlled clinical study evaluating 186 patients with poor pain control or with morphine side effect, switched to oxicodone as first line. Among 48 switchers, 41 patients (87\%) did improve when switched to an alternative opioid. It is remarkable that 37 of 
41 patients were switched to oxycodone. In a multivariate analysis the authors reported a clinical model for switching from morphine to an alternative opioid. In this study the need to switch was dependant on white cell count, weight, concomitant use of 5HT3 antiemetics, beta blockers, proton inhibitors, tumor diagnosis of the lower gastrointestinal tract and recent chemotherapy. These data seem so important because we strongly need a clinical objective model for predicting switchers.

Recently the Cochrane collaboration reported a review investigating the usefulness of opioid switching for patients with pain (Quigley 2006). The conclusion of this critical review is that there is no robust evidence base for the practice of opioid switching. However among investigated trials 13 of the 14 prospective studies reported that switching of opioids improved pain control, reduced opioid adverse effect or both. This means that switching opioids may be the only effective option for a subgroup of cancer patients despite the lack of evidence base for this strategy to counteract the imbalance between pain control and side effects. In a dedicated center for pain management, switching may be an elegant strategy for pain control. This approach needs familiarity with a range of opioids and with the use of opioid dose conversion tables previously published by the EAPC (Hanks et al 2001). The use of tables may represent a valid therapeutic option only in centers with experience in palliative care. One caveat with opioid conversion tables is that they are usually based on single doses of drugs or drugs at steady state and may not accurately represent the dose needed when titrating uncontrolled pain. The table of the relative potencies of opiates have been reasonably well established in naive patients and are not accurate in tolerant patients and using these relative potencies when switching drugs in patients highly tolerant can lead to serious problems. Clinically, it is often necessary to reduce the equivalent dose calculated from the relative potency ratios in naïve patients of the second drug by $50 \%$ or more (Pasternak 2005).

Several authors recommend decreasing the "equianalgesic" dose by $25 \%$ to $50 \%$ when switching to a new opioid to account for incomplete cross-tolerance (Ripamonti et al 1998).

\section{What is the role of other potent opioids}

Oral morphine has been recognized as the analgesic of choice in the management of cancer patients even though it is not based on proven therapeutic superiority over other options. In addition a number of patients cannot tolerate it at the doses necessary to achieve an adequate analgesia without experiencing adverse effects.

Patients who don't achieve adequate pain relief or experiencing side effects with morphine may often be successfully treated with other potent opioids. Among those, methadone, long acting oxycodone, and transdermal fentanyl have been extensively studied in controlled clinical trials.

\section{Methadone}

Methadone is a $\mu$-opioid agonist, approved for oral and intramuscular use, it also is used rectally, intravenously, subcutaneously, epidurally, and intrathecally. Methadone is a highly lipophilic molecule that is suitable for a variety of administration routes. Oral methadone has a bioavailability close to $80 \%$ compared with $20 \%-30 \%$ for morphine. Analgesia and typical opioid side effects are the result of action at the $\mu$-opioid receptor. The elimination half life is of about 22 hours. Because of its long half-life, plasma levels of methadone may take five to seven days to stabilize.

Several days are required to reach steady-state plasma levels, so monitoring should continue after the last dosage increase to detect potential overdose. Toxicity occurs more frequently in patients previously exposed to high dosages of opioids. Transition from high-dosage opioids may be difficult and needs assistance from a pain specialist.

Methadone has a number of potential advantages compared with other opioids, including morphine. Due to the low cost it could be a good choice of an opioid for firstline cancer pain treatment for low-income populations or in developing countries. In addition, methadone does not have any known active metabolites and does not undergo significant renal elimination (Bruera and Sweeny 2002). Unlike morphine, it usually is not necessary to adjust the dosage of methadone in patients with renal insufficiency.

Another potential advantage of methadone over other opioids is that this drug is a relatively potent $N$-methyl-Daspartate (NMDA) receptor antagonist. NMDA has been implicated in the development of neuropathic pain and opioid tolerance (Elliot et al 1994). One disadvantage of using methadone is that it has a long and unpredictable halflife, which can makes titration difficult to achieve (Ripamonti et al 1997, 1998). Titration might be easier and safer in patients who have not previously received strong opioids (Mercadante et al 1998).

The results of a recent randomized clinical trial did not support a relatively more effective role for methadone in patients with neuropathic pain syndromes as compared with morphine (Bruera et al 2004). Up now there is no trial 
evidence to support the proposal that methadone has a particular role in neuropathic pain of malignant origin. Methadone has a similar efficacy and a comparable side effect profile with morphine. Due to complex pharmacokinetics, its use requires expertise and experienced doctors.

\section{Oxycodone}

Oxycodone is a $\mu$-opioid receptor specific ligand with clear agonist properties, with a high oral to parenteral bioavailability, and a two-fold greater oral potency than oral morphine. Oxycodone has a more favorable pharmacokinetic profile. Its oral bioavailability is significantly higher and therefore the interindividual variation in bioavailability and expected plasma concentrations is less. CR oxycodone every 12 hours is as effective as IR oxycodone four times daily in managing moderate to severe cancer-related pain and was associated with fewer reports of adverse events (Kaplan et al 1998)

Maddocks and colleagues (1996) showed that oxycodone administered by the subcutaneous route can provide effective analgesia without significant side effects in patients with morphine-induced delirium. Two prospective studies confirmed the data (Heiskanen and Kalso 1997; Mucci-LoRusso et al 1998). Less nausea (Heiskanen and Kalso 1997) and less pruritus (Mucci-LoRusso et al 1998) have been reported with oxycodone compared with morphine.

Both CR oxycodone and $\mathrm{CR}$ morphine provide, at proper doses, pain relief for 12 hours. The onset of analgesia is faster with CR oxycodone (Curtis et al 1999). Three randomized and controlled studies have compared CR oxycodone and morphine. Two of these studies have used crossover designs (Heiskanen and Kalso 1997; Bruera et al 1998) whereas one has been a parallel group study (MucciLoRusso et al 1998). A total of 177 patients were included in these studies and $73 \%$ completed the study protocol. In the trial by Bruera and colleagues (1998), the median oxycodone/morphine dose ratio was 1:5 and the maximum was 2:3. These studies showed that oxycodone and morphine are equally efficacious. Oxycodone may be an interesting drug for pain control as first line therapy but also in the switching strategy when morphine is used as drug of choice. CR oxycodone can be used as readily as the IR formulation for titration to stable analgesia in patients with moderate to severe chronic pain due to cancer. CR oxycodone is characterized by an initial rapid absorption of $38 \%$ of the dose ( $\mathrm{t} 1 / 2$ after absorption [abs] $=37$ minutes), providing onset of analgesia within 1 hour in most patients (Mandema et al 1996; Salzman et al 1999).

\section{Transdermal fentanyl}

Fentanyl is a lipid soluble synthetic opioid. The high lipid solubility facilitate the absorption though the skin. The transdermal delivery system provides a consistent rate of fentanyl to the microcirculation of the skin and the steady state rate is achieved at 72 hours. This type of delivery is applied every 3 days. Transdermal fentanyl was compared with CR morphine in patients with terminal cancer pain (Ahmedzai and Brooks 1997). No significant differences in analgesic efficacy or adverse events were observed between the two groups of patients. Due to limitations of patch size small increment of dose are not possible. In addition the long half life does not allow rapid change of dose for patients with unstable pain.

Recently Ripamonti and colleagues (2006) showed that, in Italy, there is a trend to use fentanyl patch as first-choice strong opioid in cancer patients in situations such as titration phase, in the presence of unstable pain, and in the absence of dysphagia or gastrointestinal symptoms where the use of oral morphine is not contraindicated. According to the EAPC (Hanks et al 2001) transdermal fentanyl is reserved for patients who require stable opioid doses or for patients unable to take oral morphine as an alternative to the subcutaneous route.

\section{Conclusions}

Cancer pain is often suboptimally managed. The underestimation and undertreatment of cancer pain continues to be a problem despite the availability of consensus-based guidelines. A holistic approach including an appropriate use of opioids may improve pain control in most cancer patients, particularly for those with advanced disease.

\section{References}

Ahmedzai S, Brooks D. 1997. Transdermal fentanyl versus sustainedrelease oral morphine in cancer pain: preference, efficacy, and quality of life. The TTS-Fentanyl Comparative Trial Group. J Pain Symptom Manage, 13:254-61

Ashby MA, Martin P, Jackson KA. 1999. Opioid substitution to reduce adverse effects in cancer pain management. Med J Aust, 170:68-71.

Battaglia J, Moss S, Rush J, et al. 1997. Haloperidol, lorazepam, or both for psychotic agitation? A multicenter, prospective, double blind, emergency department study. Am J Emerg Med, 15:335-40.

Bruera E, MacMillian K, Hanson J, et al. 1989. The cognitive effects of the administration of arcotics analgesics in patients with cancer pain. Pain, 39:13-16. 
Bruera E, Miller L, McCallion J, et al. 1992. Cognitive failure in patients with terminal cancer: a prospective study. J Pain Symptom Manage, 7:192-5.

Bruera E, Palmer JL, Bosnjak S, et al. 2004. Methadone versus morphine as a first-line strong opioid for cancer pain: a randomized, doubleblind study. J Clin Oncol, 1:185-92.

Bruera E, Sweeney C. 2002. Methadone use in cancer patients with pain: a review. J Palliat Med, 5:127-38.

Cherny N, Ripamonti C, Pereira J, et al. 2001. Strategies to manage the adverse effects of oral morphine: an evidence-based report. J Clin Oncol, 19:2542-54.

Cleeland CS, Portenoy RK, Rue M, et al. 2005. Does an oral analgesic protocol improve pain control for patients with cancer? An intergroup study coordinated by the Eastern Cooperative Oncology Group. Ann Oncol, 16:972-80.

Curtis GB, Johnson GH, Clark P, et al. 1999. Relative potency of controlled-release oxycodone and controlled-release morphine in a postoperative pain model. Eur J Clin Pharmacol, 55:425-9.

Davis MP, Walsh D, Lagman R, et al. 2005. Controversies in pharmacotherapy of pain management. Lancet Oncol, 6:696-704.

De Conno F, Craceni A, Martini C, et al. 1991. Hyperalgesia and myoclonus with intrathecal infusion of high-dose morphine. Pain, 47:337-9.

Du Pen SL, Du Pen AR, Polissar N, et al. 1999. Implementing guidelines for cancer pain management: results of a randomized controlled clinical trial. J Clin Oncol, 17:361-70.

Bruera E, Belzile M, Fainsinger R, et al. 1998. Randomized, double-blind, cross-over trial comparing safety and efficacy of oral controlled-release morphine in patients with cancer pain. $J$ Clin Oncol, 16:3222-9.

Davis MP, Walsh D, Lagman R, et al. 2003. Randomized clinical trial of an implantable drug delivery system J Clin Oncol, 21:2800-1.

Eisele JH, Grigsby EJ, Dea G, et al. 1992. Clonazepam treatment of myoclonic contractions associated with hidose opioids: case report. Pain, 49:231-2.

Elliott K, Minami N, Kolesnikov Y, et al. 1994. The NMDA receptor antagonists, LY274614 and MK-801, and the nitric oxide synthase inhibitor, NG-nitro-L-arginine, attenuate analgesic tolerance to the mu-opioid morphine but not to the kappa opioids. Pain, 56:69-75.

Gordon DB, Dahl JL, Miaskowski C, et al. 2005. American pain society recommendations for improving the quality of acute and cancer pain management: American Pain Society Quality of Care Task Force. Arch Intern Med, 165:1574-80.

Hanks GW, Conno F, Cherny N, et al. 2001. Morphine and alternative opioids in cancer pain: the EAPC recommendations. $\mathrm{Br} J$ Cancer, 84:587-93.

Heiskanen T, Kalso E. 1997. Controlled-release oxycodone and morphine in cancer related pain, Pain, 73:37-45.

Kalso E, Heiskanen T, Rantio M, et al. 1996. Epidural and subcutaneous morphine in the management of cancer pain: a double-blind crossover study. Pain, 67:443-9.

Kaplan R, Parris WC, Citron ML, et al. 1998. Comparison of controlledrelease and immediate-release oxycodone tablets in patients with cancer pain. J Clin Oncol, 16:3230-7.

Levy MH, Samuel TA. 2005. Management of cancer pain. Semin Oncol, 32:179-93.

Maddocks I, Somogyi A, Abbott F, et al. 1996. Attenuation of morphine induced delirium in palliative care by substitution with infusion oxycodone. J Pain Symptom Manage, 12:182-9.

Mandema JW, Kaiko RF, Oshlack B, et al. 1996. Characterization and validation of a pharmacokinetic model for controlled-release oxycodone. Br J Clin Pharmacol, 42:747-56.

McNicol E, Strassels SA, Goudas L, et al. 2005. NSAIDs or paracetamol, alone or combined with opioids, for cancer pain. Cochrane Database Syst Rev, 2:CD005180.
Mercadante S. 1999. Opioid rotation for cancer pain: Rationale and clinical aspects. Cancer, 86:1856-66.

Mercadante S, Casuccio A, Agnello A, et al. 1998. Morphine versus methadone in the pain treatment of advanced cancer patients followed up at home. J Clin Oncol, 16:3656-61.

Mercadante S, Casaccio A, Fulfaro F, et al. 2001. Switching from morphine to methadone to improve analgesia and tolerability in cancer patients: a prospective study. J Clin Oncol, 19:2898-904.

Mucci-LoRusso P, Berman BS, Silberstein PT, et al. 1998. Controlledrelease oxycodone compared with controlled-release morphine in the treatment of cancer pain. A randomized, double-blind, parallel-group study. Eur J Pain, 2:239-49.

Obeso JA. 1995. Therapy of myoclonus. Clin Neurosci, 3:253-7.

Paix A, Coleman A, Lees J, et al. 1995. Subcutaneous fentanyl and sufentanil infusion substitution for morphine intolerance in cancer pain management. Pain, 63:263-9.

Pasternak GW. 2005. Molecular biology of opioid analgesia. J Pain Symptom Manage, 29:S2-9.

Quigley C. 2006. Opioid switching to improve pain relief and drug tolerability. Cochrane Database Syst Rev, 2.

Riley J, Ross JR, Rutter D, et al. 2006. No pain relief from morphine? Individual variation in sensitivity to morphine and the need to switch to an alternative opioid in cancer patients. Supp Care Cancer, 14:5664.

Ripamonti C, Brunelli C. 2003. Randomized clinical trial of an implantable drug delivery system compared with comprehensive medical management for refractory cancer pain: impact on pain, drug-related toxicity, and survival. J Clin Oncol, 21:2801-2.

Ripamonti C, Fagnoni E, Campa T, et al. 2006. Is the use of transdermal fentanyl inappropriate according to the WHO guidelines and the EAPC recommendations? A study of cancer patients in Italy. Supp Care Cancer, 14:400-7.

Ripamonti C, Groff L, Brunelli C, et al. 1998. Switching from morphine to oral methadone in treating cancer pain: what is the equianalgesic dose ratio? J Clin Oncol, 16:3216-21.

Ripamonti C, Zecca E, Bruera E. 1997. An update on the clinical use of methadone for cancer pain. Pain, 70:109-15.

Salzman RT, Roberts MS, Wild J, et al. 1999. Can a controlled-release oral dose form of oxycodone be used as readily as an immediaterelease form for the purpose of titrating to stable pain control? J Pain Symptom Manage, 18:271-9.

Sjogren P, Banning A. 1989. Pain, sedation and reaction time during longterm treatment of cancer patients with oral and epidural opioids. Pain, 39:5-11.

Smith TJ, Coyne PJ, Staats PS, et al. 2005. An implantable drug delivery system (IDDS) for refractory cancer pain provides sustained pain control, less drug-related toxicity, and possibly better survival compared with comprehensive medical management (CMM). Ann Oncol, 16:825-33.

Smith TJ, Staats PS, Deer T, et al. 2002. Randomized clinical trial of an implantable drug delivery system compared with comprehensive medical management for refractory cancer pain: impact on pain, drugrelated toxicity, and survival. J Clin Oncol, 19:4040-9.

Trowbridge R, Dugan W, Jay SJ, et al. 1997. Determining the effectiveness of a clinical-practice intervention in improving the control of pain in outpatients with cancer. Acad Med, 72:798-800.

Vainio A, Tigerstedt I. 1988. Opioid treatment for radiating cancer pain: oral administration vs. epidural techniques. Acta Anaesthesiol Scand, 32:179-85.

Walsh TD, MacDonald N, Bruera E, et al. 1992. A controlled study of sustained release morphine sulphate tablets in chronic pain from advanced cancer Am J Clin Oncol, 15:268-72. 
